\title{
Multi-Sensor Fusion of the Foreleg Mechanism for Wheelchair Climbing-Stairs
}

\author{
Dongxing Cao*, Weixiang Di, Qiang Wang and Minfei Li \\ Hebei University of Technology, School of Mechanical Engineering, China \\ ${ }^{*}$ Corresponding author
}

\begin{abstract}
The foreleg mechanism plays an important role to carry out climbing stairs and crossing obstacles for wheelchair climbing-stairs. Multi-sensors are placed on different site of the foreleg mechanism, and their measurement values timely decide and control the foreleg position and posture change. The driver module includes multi-sensors, relay operation, and motor fuzzy control, and the control system module includes manual and automatic control. Multi-machine communication is realized to exchange data between the top computer and MCU. An effective multi-sensor fusion algorithm is proposed to describe the process of the foreleg motion. Error factors of influencing sensor measurement are analyzed, and the experimental result shows the effectiveness of the multi-sensors fusion algorithm.
\end{abstract}

Keywords-foreleg; multi-sensors; wheelchair climbing-stair

\section{INTRODUCTION}

The wheelchair climbing-stairs, as a kind of service robots, plays an important role for the elderly and disabled going outside. However, the foreleg mechanism motion is the key to achieve the success of climbing-stairs. In order to effectively realize climbing act, an intelligence control method is proposed to describe the process of wheelchair robot coordination work, in which the foreleg mechanism can feel its environment and make response to action [1-2]. Multi-sensors are installed on the foreleg, and used to environmental perception. Several sensors are adopted to measure the posture of the foreleg. They can timely follow the change of distance and angular which controls the motional state of the foreleg. In addition, an effective algorithm is presented to model wheelchair climbing stairs and their route planning. A multi sensor feedback system was developed to assist monitor wheelchair robot act.

\section{MOTION REQUIREMENTS OF FORELEG}

The foreleg is a key mechanism of wheelchair climbingstairs. It decides whether the wheelchair robot is able to successfully climb. When the wheelchair robot begins to climb stairs, the foreleg will need to rise and fall motion for fitting different steps changes. Both forelegs constantly need to move up and down. At the same tome, each foreleg has two motion freedom degrees, that is, one is the pendulum motion, and another is uplift motion [3]. Their motion is coupling, that is, one freedom degree is to affect or limit another freedom degree. This needs to obtain the motional range of each freedom degree. These ranges accordingly limit the foreleg mechanism motion as shown in Figure 1. Thus, the foreleg act needs to meet the following requirements [4].
- two forelegs lifting, swinging, and stretching at the same time.

- two foreleg actions avoiding within dead range.

- two foreleg motion space is as large as possible.

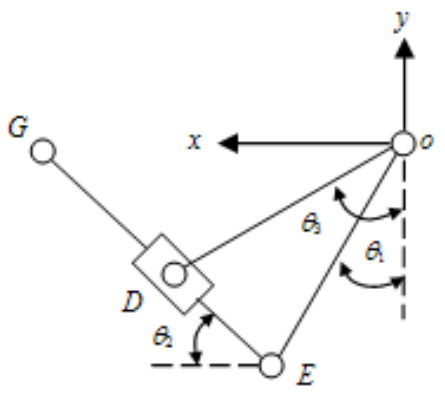

FIGURE I. THE FORELEG MECHANISM

\section{Muti-SENSOR Placement}

The sensor install location depends on the foreleg structure. As the foreleg has three freedom degrees, at least three sensors are installed on the foreleg. Here three sensor locations are given to measure different act changes.

\section{A. Distance Measurement}

The ultrasonic sensor is used to measuring the distance of the foreleg lifting. It will be installed on the fixed platform of the foreleg, which the sensor is fixed relative to the foreleg, and the platform is fixed on the frame of wheelchair robot. When the foreleg moves up and down, the sensor can measure the distance change. Also another ultrasonic sensor is installed on the bar linkage of the foreleg, and it measures the distance change of slider movement compared with the bar linkage as shown in Figure 2. Thereby, it can realize the measurement of swinging motion [5]. 


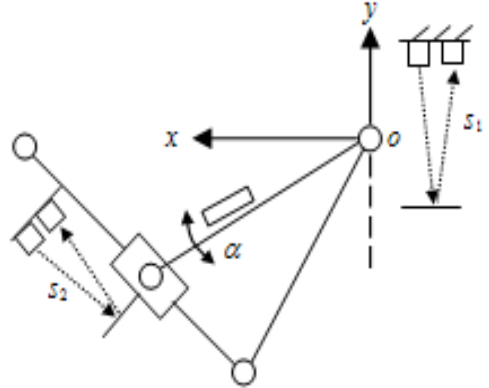

FIGURE II. THE LOCATION GRAPH OF DIFFERENT SENSORS

\section{B. Angle Measurement}

The angular transducer is an effective means to measure position and posture change of the foreleg, which is fixed on the guide rod, and it can measure the angular of the foreleg uplifting as shown in Figure 2.

The output of gyroscope is angular velocity, and after the integration the angle is obtained and it exists in cumulative error. On the other hand, the output of an accelerometer is acceleration, and not appears cumulative error. However, it is influenced by the vibration from device motion. Both device combinations can accurately measure angle as shown in Figure 3.

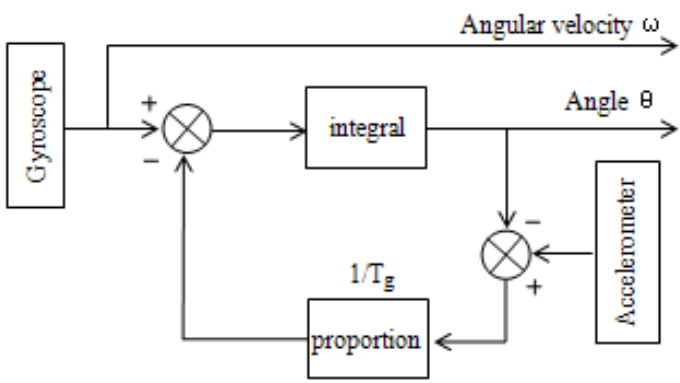

FIGURE III. GRAVITY ACCELEROMETER CORRECTING ANGLE DEVIATION

\section{Sensor Istallation Error Analysis}

The reflection surface of ultrasonic sensor needs to be smooth so that the sending acoustic wave can be received successfully. When reflection surface is not slippery, or vibrancy, or declining, echo signal can't receive correctly. Therefore, when the reflection surface is installed, it needs to be vertical wave propagation direction.

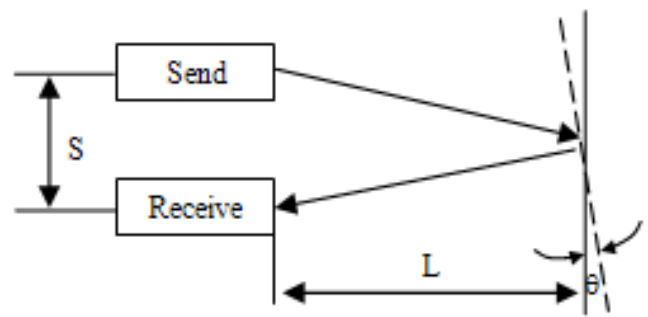

FIGURE IV. THE PRINCIPLE OF THE ULTRASONIC SENSORS
At zero centigrade, the velocity of ultrasonic wave propagation is $331.4 \mathrm{~m} / \mathrm{s}$. As the temperature changes, the speed of ultrasonic propagation also changes. Thus, considering the influence of temperature on the measurement accuracy, the velocity of ultrasonic wave propagation is as follows.

$$
v \approx 331.4+0.607 \mathrm{~T}
$$

Where, $\mathrm{T}$ stands for the temperature.

The distance $(d)$ of wave propagation is

$$
d=v \cdot \mathrm{t}
$$

Thus,

$$
L=0.5\left(d^{2}-s^{2}\right)^{1 / 2}
$$

When $d>>s$, then

$$
L \approx 0.5(331.4+0.607 \mathrm{~T}) \mathrm{t}
$$

When the reflection surface has a tilt angle $\theta$, the travel of wave propagation will become longer. Then the distance of measurement become larger. In such doing, when the ultrasonic sensor receives signal, it has a delay problem. And it influences the measuring distance accuracy. In addition, the tilt angle $\theta$ depends on screw nut drive mechanism manufacturing accuracy, and the foreleg mechanism positioning errors, etc.

In order to reduce the influence of the above-mentioned factors on the accuracy of measurement, we need to add some delay in software, for example, $0.05 \mathrm{~ms}$ can be easily improved.

\section{CONTROL SyStem DESIGN OF ForELEG}

The foreleg mechanisms locate in the left front and right front of the wheelchair climbing-stairs. They are symmetrically mounted on both sides of the wheelchair robot [6]. Their framework of control system is presented in Figure 5. And it includes three main modules in the following. 


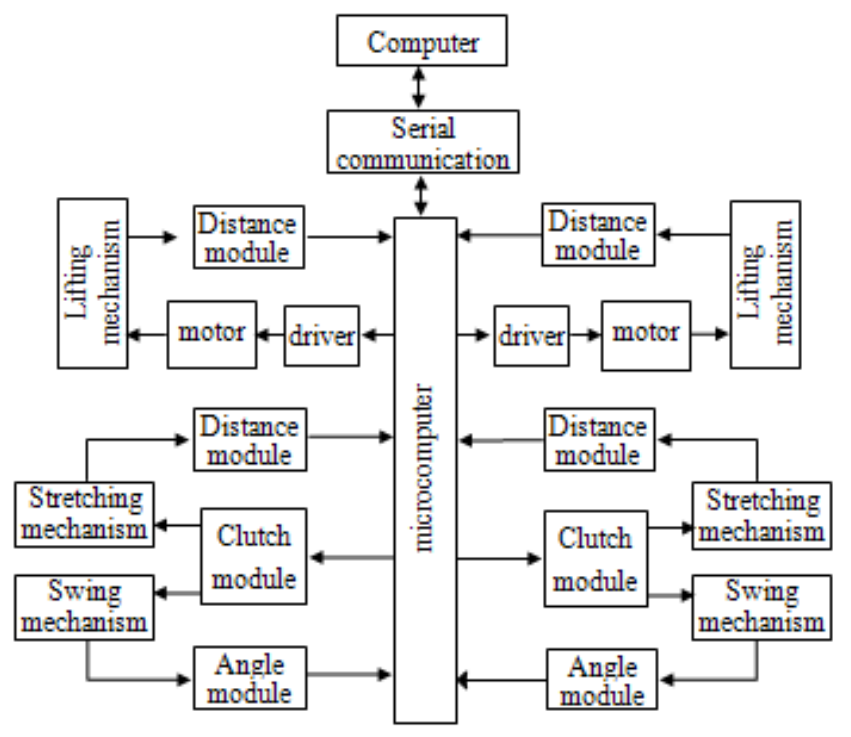

FIGURE V. THE FRAMEWORK OF THE FORELEG CONTROL SYSTEM

\section{A. Lifting Mechanism}

The lifting mechanism, as the foreleg platform, has an independent driving motor, and receives commands from top computer to implement up and down motion. Both side lifting mechanisms need to maintain the motional synchronism and real time. If they have non synchronous motion in the process of climbing-stairs, the wheelchair robot will lead to rollover. Therefore, the lifting mechanism is required to obtain motion control accurately, and both need real time keeping in the same level.

\section{B. Posture Adjusting Mechanism}

The swinging component and stretching component consist of two freedom degree posture adjusting mechanism. As their drivers share a motor, they form an under-actuated mechanism. Thus, two mechanism motion exist in some coupling relation, not an independent each other. Angle measuring module is used to closed loop feedback control of swinging mechanism as shown in Figure 5. Also the distance measuring module is used to a closed loop feedback control of stretching mechanism.

\section{Dead Rang of Foreleg Motion}

As the position and posture adjusting mechanism is stronger coupling, their movement is limited the dead point [3]. Considering some errors from manufacturing and assembly, the dead point will become a range. Therefore, the foreleg mechanism motion should avoid the dead range, and select the region with good force characteristics. Figure 6 presents a boundary line with the dead range. Right side of the coupling boundary line is the prohibition of running areas. It guarantees the safety of the foreleg motion. Also the foreleg correctly runs between distance top boundary line and distance lower boundary line, and works right side of angle boundary line and left side of coupling boundary line.

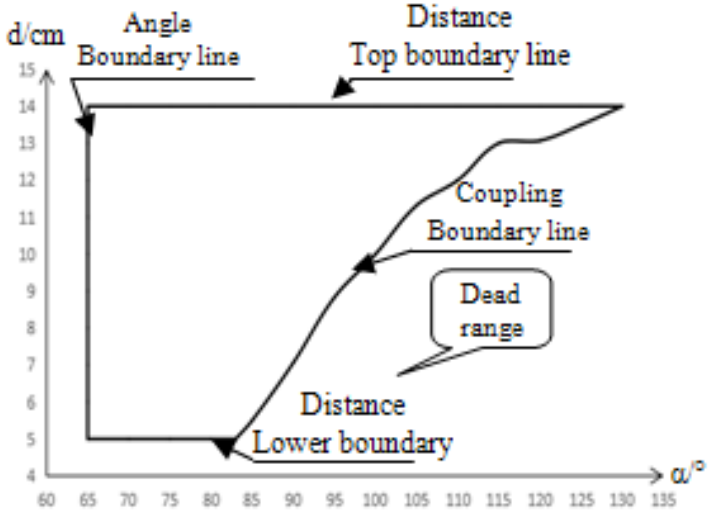

FIGURE VI. THE ANALYSIS OF THE DEAD RANGE

\section{CONTROL ALGORITHM IMPROVEMENT}

As the foreleg motion needs to maintain the balance of wheelchair robot, lifting mechanism and position and posture adjusting mechanism should correctly move and accurately measure the distance and angle. This needs that the value from each sensor is accuracy, and an effective algorithm can be implemented to ensure stable motion.

\section{A. Lifting Mechanism Control}

Both side lifting mechanism movements are independent. Each is driven by itself motor. Object distance is from top computer sending the specified height. At first, left side lifting distance is considered active motion, while right lifting is viewed to passive follow motion. Secondly, right side follows left side motion according to fuzzy control algorithm. If both distance difference exists, they will approximate set value in the light of fuzzy control as shown in Figure 8. Only when their distance difference reach precision requirement, the lifting mechanism motion will stop movement.

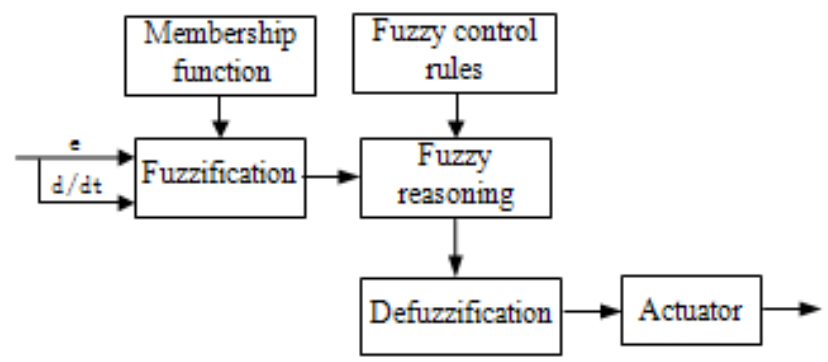

FIGURE VII. THE LIFTING MECHANISM CONTROL

\section{B. Position and Posture Mechanism Control}

Also right side position posture mechanism still follows the left movement as a reference. When the distance difference is not satisfied with the set value, their motion adjusting will depends on fuzzy control. The operation will stop until the precision requirement [7]. In addition, angle adjusting still follows the left angle motion as a reference. Only when their angle difference reach precision requirement, the swinging mechanism motion will stop work as shown in Figure 8. 


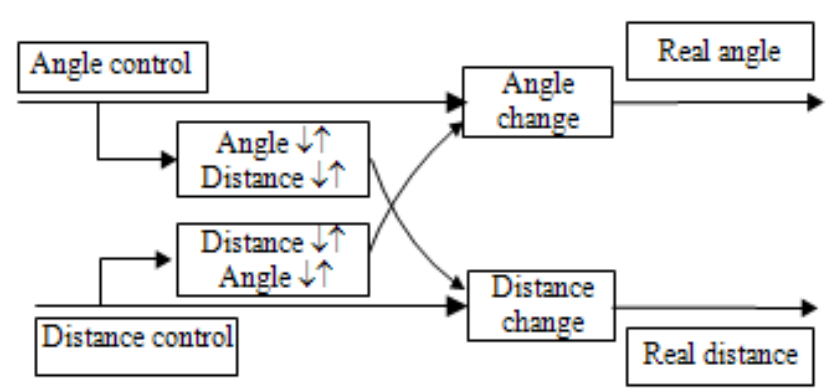

FIGURE VIII. POSITION AND POSTURE MECHANISM CONTROL

\section{EXPERIMENT RESULT ANALYSIS}

The foreleg control system includes two work modes, that is, manual control and automatic control. The former is used to familiar with the foreleg mechanism motion characteritic, and debugging the foreleg mechanism act to meet the requirements of action. And test multi-communications and exchange data between the top computer and MCU. The latter is used to test the ability of left and right foreleg coordinate motion, and fast response characteristic and mobility flexibility. At the same time, the foreleg control system still provides the monitoring and alarm function. It can flexibly implement the foreleg uplifting, swinging, and stretching movement. Table 1 presents the data from senor measuring uplifting and swinging motion. The tested distance data give the relationship between time and distance, and accordingly calculate the speed of the foreleg response. Also tested angle data is shown in Table 1 yet. In fact, the uplifting motion of left and right foreleg is synchronous. Only relative synchronization has no absolute synchronization for both side forelegs. After several experiments we will obtain stable and reliable data. At the same time, we still obtain fuzzy control rule table [8]. Figure 10 presents uplifting motion fuzzy control curve (solid line) and PID control curve (dash line) comparison. Fuzzy control curve is gradually approaching the set value, however, when it is near the set value, there is an up and down oscillation process for PID control curve. Figure 11 presents angle change fuzzy control curve (solid line) and PID control curve (dash line) comparison. Also, in the process of angle control, there is a similar change. The only difference is upper and lower oscillations to be delayed.

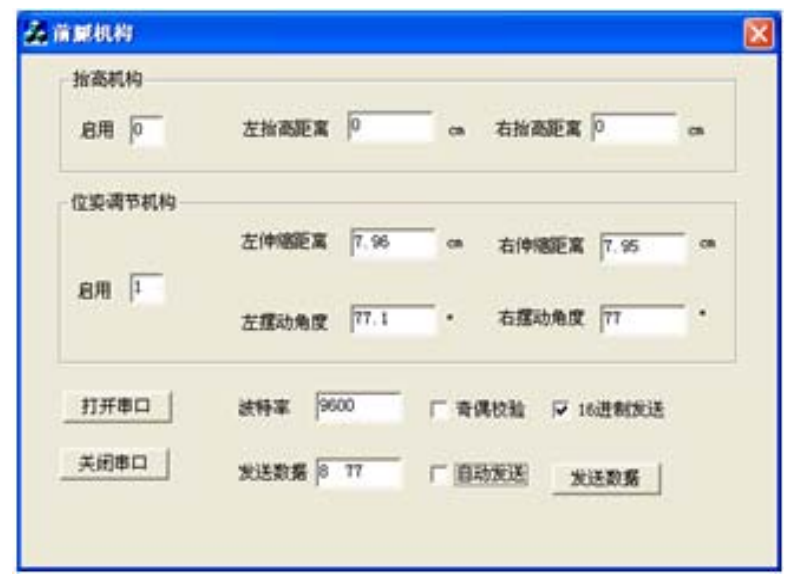

FIGURE IX. THE POSTURE MECHANISM CONTROL
TABLE I. UPLIFTING AND SWINGING DATA

\begin{tabular}{|c|c|c|c|c|c|c|c|c|}
\hline \multirow{2}{*}{ 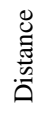 } & 0 & 0.2 & 0.4 & 0.8 & 0.9 & 1 & 1.1 & 1.2 \\
\hline & 5 & 5.4 & 5.8 & 6.96 & 6.8 & 7 & 7.08 & 7.16 \\
\hline \multirow{2}{*}{$\begin{array}{l}\frac{0}{00} \\
\frac{8}{4}\end{array}$} & 0 & 0.2 & 0.6 & 1 & 1.2 & 1.6 & 1.8 & 2 \\
\hline & 70 & 70.4 & 71.2 & 72 & 72.4 & 73.2 & 73.6 & 74 \\
\hline
\end{tabular}

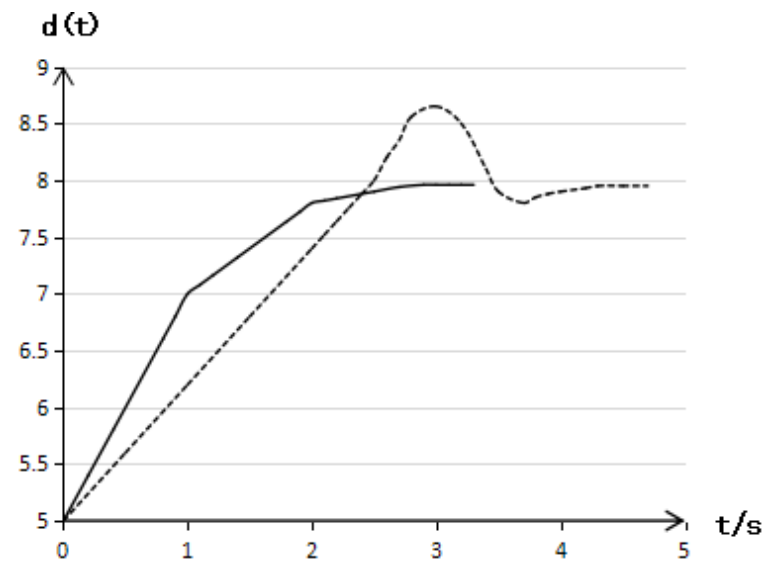

FIGURE X. FUZZY AND PID CONTROL CURVES OF LIFTING a (t)

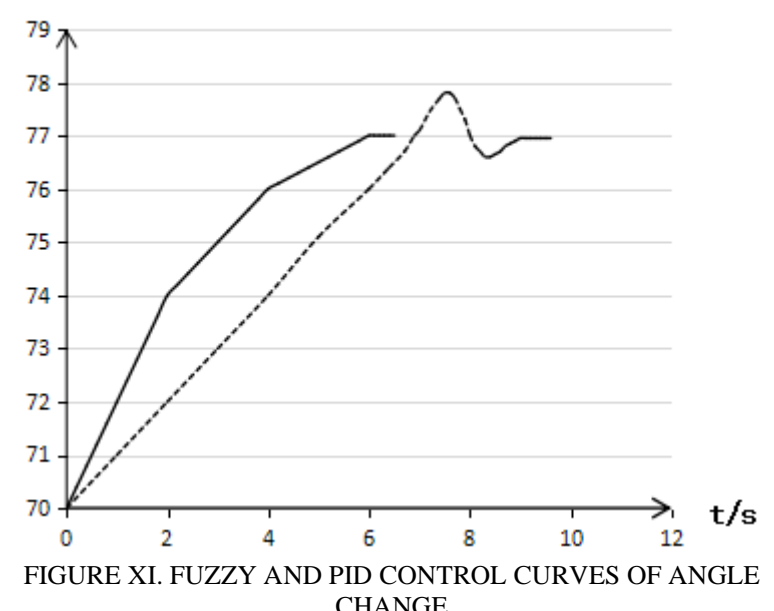

ACKNOWLEDGMENT

This research was financially supported by NSF of China under Grant No. 51275152 and NSF of Hebei Province under Grant No. 2018202114.

\section{REFERENCES}

[1] B Rodrigo Antonio Marques, P Marcelo, R Luis Paulo, etc. Intellwheels: Modular development platform for intelligent wheelchairs. Journal of Rehabilitation Research and Development, 2011, 48(9): 1061-1076

[2] Dongxing Cao, Zhanwei Li, Hui Li, Baoling Zong Port-based ontology behavioral graph modeling for climbing robot route planning, WIT Transactions on Engineering Sciences, 2015, Vol.102: 117-123.

[3] Bao-ling Zong, Dong-xing Cao A foreleg mechanism design of wheelchair robot on port modeling, 2015 International Conference on Mechanical Science and Mechanical Design, Dec. 12, 2015, Changsha. 
[4] D.X. Cao, Q. L. Jia, J. Yang.Port-based description of functional modeling for product conceptual design. Journal of Advanced Manufacturing Systems, 2008,7(1): 101-105

[5] Sinha, R.; Paredis, C.J.J.; and Khosla, P.K. Integration of Mechanical CAD and VHDL-AMS Behavioral Modeling, proceedings of IEEE/ACM BMAS 2000, Orlando, FL, USA.

[6] D.X. Cao, N.H. Zhu, C.X. Cui, et al. An agent-based approach to guiding the conceptual design of mechanical products, International Journal of Production Research, 2008, 46 (9): 2381-2396

[7] Liu Shan-shan, Cao Dong-xing, Wang Chao Research on control strategy of chassis mechanism of climbing wheelchair based on multisensors Transducer and Micerosystem Technology, 2018, 37(1): 70-78.

[8] Holly Moore. MATLAB for Engineers Second Edition[M]. Publishing House of Electronics Industry, 2010. 